\title{
AN UNUSUAL CAUSE OF CARPAL TUNNEL SYNDROME
}

\author{
A Case of Thrombosis of the Median Artery
}

\author{
Ian T. Jackson and John C. Campbell, Glasgow, Scotland
}

From the West of Scotland Plastic Surgery Service, Canniesburn Hospital, Glasgow, and the Institute of Pathology, Royal Infirmary, Glasgow'

Compression of the median nerve as it lies in the carpal tunnel can be caused by many lesions. Entin (1968) grouped them into three categories: those reducing the capacity of the tunnel, those increasing the volume of its contents, and those that form part of a systemic condition.

Recently a case of the syndrome presented which fell into the second grouping: the cause was unusual and a search of the literature has failed to reveal any reports of a similar condition.

\section{CASE REPORT}

A man aged thirty-seven years was admitted for excision of a pleomorphic salivary adenoma of the soft palate. His occupation, cooper, is extremely heavy, calling for almost constant power movements of upper limbs and hands. During his general examination he

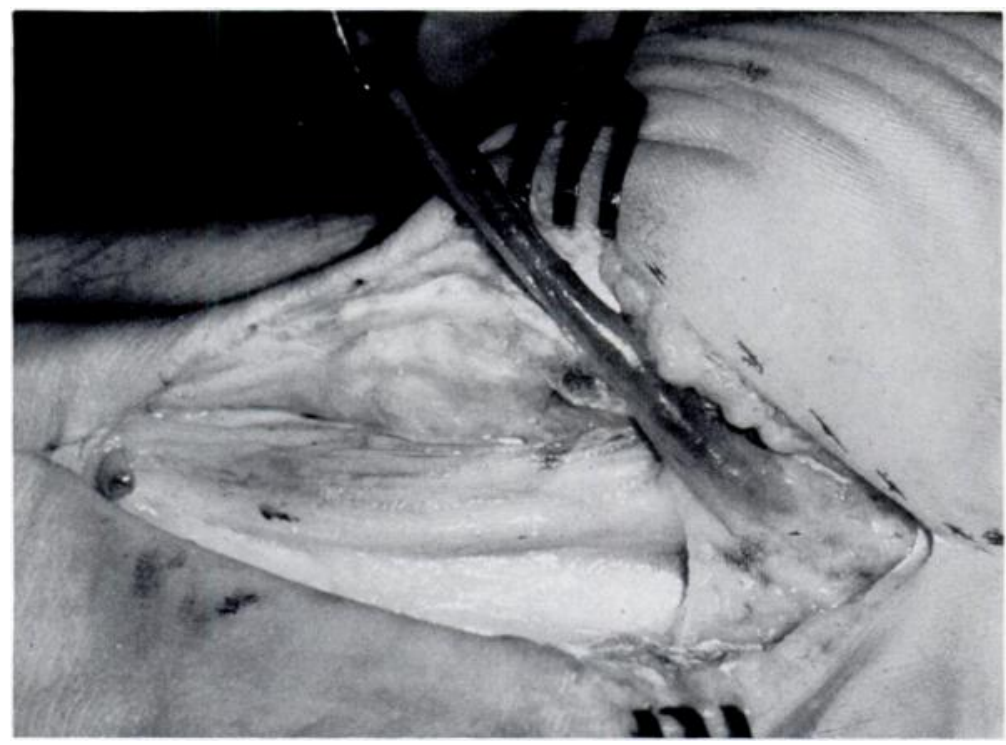

Fig. 1

After division of the carpal ligament the dilated artery has been dissected off the median nerve. Note the wide exposure which should be used in exploration for median nerve compression.

volunteered that six weeks previously he had begun to suffer pain in the left hand. He could remember the exact day when he first noticed it and was certain that its onset had not been related to injury or to unusual wrist movement. The pain was confined to the radial side of the palm of the hand and extended into the radial three and a hâlf fingers. Its intensity varied from slight paraesthesia to severe pain, the latter interfering with his ability to perform his work and keeping him awake at night, at which time it was most intense. He was able to elicit tenderness by pressing over the carpal tunnel region. These symptoms had gradually 
become worse and had reached a fairly static level at the time of examination. His general health was good.

On examination there was a fullness on the volar aspect of the wrist immediately proximal and distal to the carpal ligament. In addition there was a radial to ulnar convexity over the carpal ligament as compared to the normal concavity on the other wrist. Pressure over this area caused local pain, and Tinel's sign was positive. There was evident wasting of the thenar muscles. General examination of the patient and radiographs of the wrist revealed no abnormality.

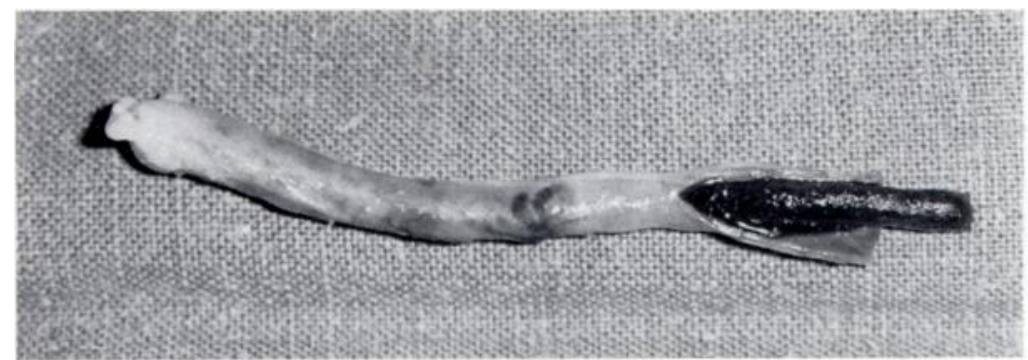

FIG. 2

The dilated artery has been opened, showing some thickening of its wall. The surface of the thrombus shows a granularity suggesting that it is undergoing organisation, but it is not yet strongly adherent to the vessel wall.

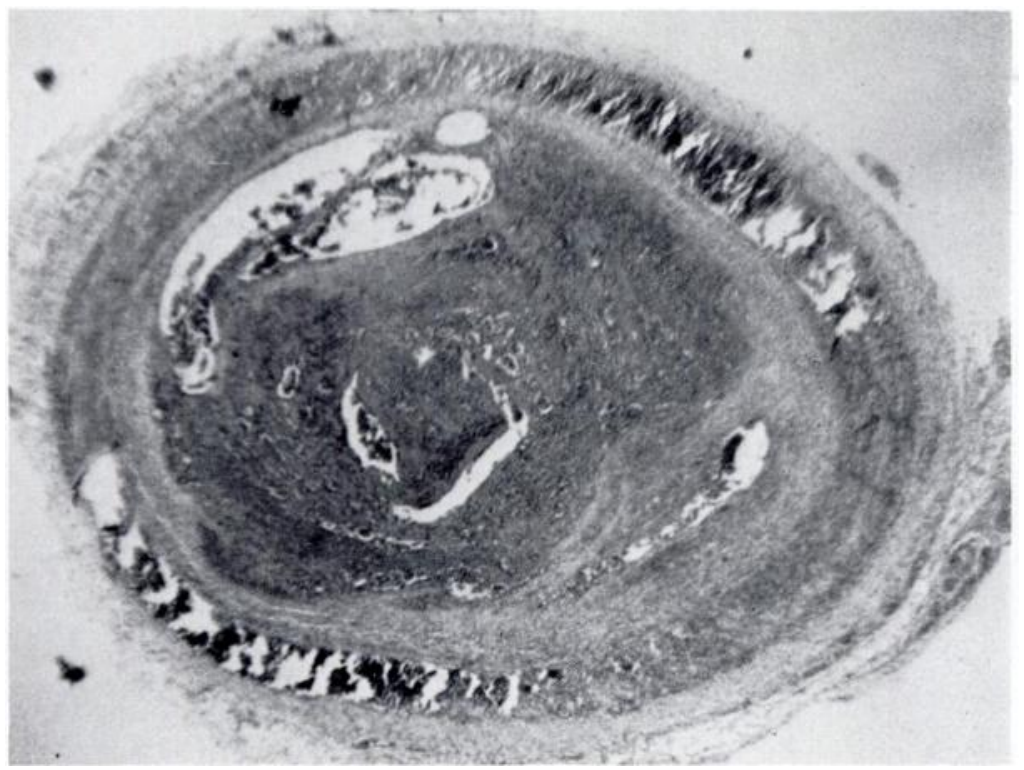

Fig. 3

Photomicrograph of a small muscular artery containing an organising thrombus. (Haematoxylin and eosin.)

Operation and progress-The carpal tunnel was explored through a wide exposure, and on division of the carpal ligament a large vessel was encountered lying on top of the median nerve (Fig. 1). It extended over a distance of 5 centimetres and was 3 millimetres in diameter. The position of this vessel suggested that it could be the median artery. The affected area was resected and was found to contain fairly recent blood clot (Fig. 2).

The symptoms were no longer present on the morning after operation. This state of affairs has continued and the patient has returned to work.

VOL. 52 B, NO. 2, MAY 1970 
Histopathology-The excised piece of the blood vessel contained a fairly recent thrombus, from which the vessel wall could be stripped without much difficulty. Microscopic examination showed several small areas of intimal hyperplasia but atheromatous changes were minimal (Fig. 3). The media contained a substantial amount of collagen but dense bands of normal smooth muscle cells were also prominent. There was no evidence of medial calcification or necrosis. A well defined internal elastic lamina was present in some areas, showing small ruptures and occasional reduplication. The appearances were those of a small muscular artery.

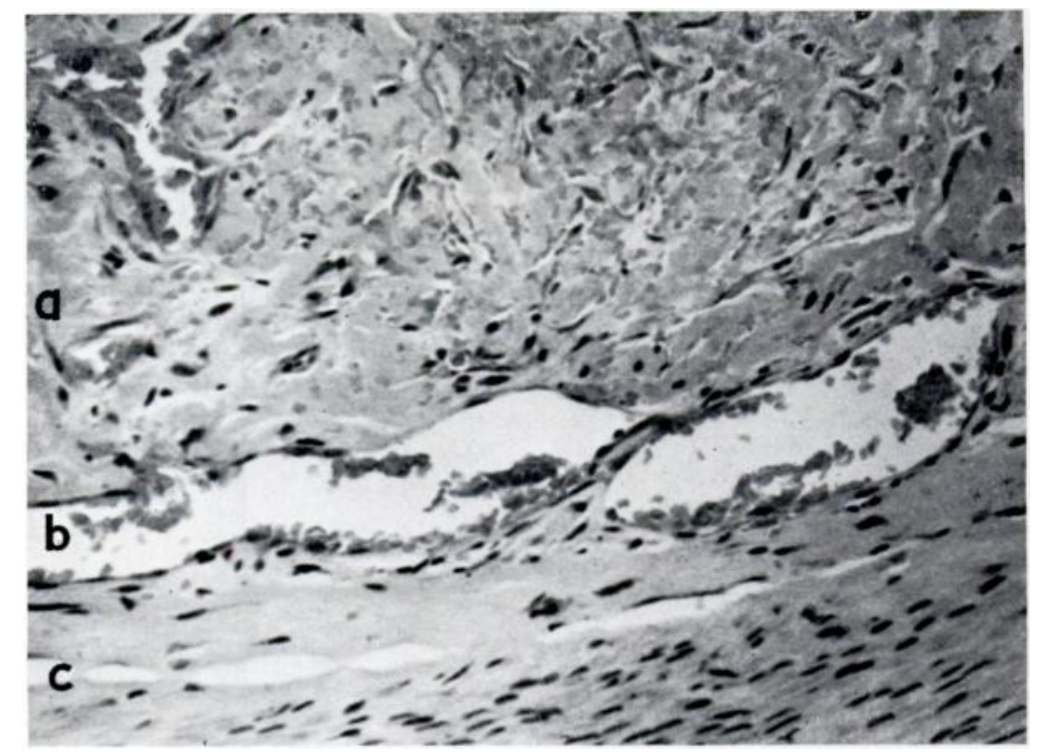

FIG. 4

Photomicrograph showing (a) thrombus with advanced organisation, (b) recanalisation, and (c) media. (Haematoxylin and eosin.)

The adventitia showed marked chronic inflammatory changes with focal lymphatic aggregates, some in relation to the vasa vasorum which were very prominent and showed slight mural thickening. Eosinophils were numerous in some areas, with occasional plasma cells and macrophages, but giant cells were not seen and occlusion of the vasa vasorum was not observed. Haemosiderin deposition in the intima and the adventitia was very marked and mild perineural fibrosis was present.

In the centre of the blood vessel there was a thrombus of recent origin in which well preserved red cells were present. Towards the intima, however, there were large areas where organisation was advanced and good recanalisation was present (Fig. 4). These appearances together with the amount of haemosiderin deposition would indicate that there was a new thrombotic episode in a former recanalising thrombus compatible with a period of six weeks' duration.

Histologically the lesion did not fit well into any of the recognised categories of thrombotic disorders. Some areas resembled thromboangiitis obliterans but the absence of giant cells and of damage to the media strongly opposed this diagnosis. No explanation could be offered for the number of eosinophils present.

\section{DISCUSSION}

There are three points of interest about this case. Firstly it is an unusual cause of median nerve compression within the carpal tunnel. Secondly it is possible that on organisation of 
the blood clot the symptoms may have gradually resolved, and had the patient not been having operation for the palatal tumour then this lesion might not have been uncovered. Thus, this may be a more common cause of the occasional carpal tunnel syndrome which resolves spontaneously.

Lastly, it is of some interest to attempt to postulate a cause for this. Dilation of the vessel within the canal with thrombosis is difficult to explain: it does suggest that the dilation led to stasis which in turn led to thrombosis, because had the reverse occurred then dilation outwith the canal would be expected. At operation it was thought that there would be a vessel wall lesion to account for this, but this was not confirmed by histological examination. Examination of the patient has failed to reveal any other vascular lesions or related symptomatology. Perhaps the most likely explanation is that of recurrent slight occupational trauma associated with the patient's heavy job and so habitual as to be unnoticed by him.

This case does emphasise that in most instances median nerve compression calls for early surgical treatment. The operation is simple, usually being performed as an out-patient under regional anaesthesia. Good exposure is essential since the cause of the symptoms is not always one which can be relieved by simple division of the carpal ligament. It may be, as we have suggested in this case, that the symptoms will resolve without treatment, but several painful weeks may pass before this satisfactory conclusion results.

\section{SUMMARY}

An unusual case of median nerve compression within the carpal tunnel ascribed to thrombosis in a dilated artery is described.

\section{REFERENCE}

Entin, M. A. (1968): Carpal Tunnel Syndrome and Its Variants. Surgical Clinics of North America, 48, 1097. 